\title{
Fatores comportamentais de risco para ocorrência de obesidade: Avaliação em escolas da rede municipal de ensino na Amazônia Paraense
}

\author{
Behavioral risk factors for occurrence of obesity: Evaluation in schools of the municipal education \\ network in the Paraense Amazon
}

Factores de riesgo conductuales para la ocurrencia de obesidad: Evaluación en escuelas municipales de la región amazónica de Pará Universidade do Estado do Pará, Brasil E-mail: susanicruzsousa@ hotmail.com

Tainara Silva Thomáz

ORCID: https://orcid.org/0000-0001-5080-745X Universidade do Estado do Pará, Brasil E-mail: tainarathomaz97@gmail.com

Adjanny Estela Santos de Souza ORCID: https://orcid.org/0000-0001-9054-4535 Universidade do Estado do Pará, Brasil E-mail: adjannyestela@hotmail.com

Lívia de Aguiar Valentim ORCID: https://orcid.org/0000-0003-4255-8988 Universidade do Estado do Pará, Brasil

E-mail: livia.valentim@uepa.br

Albino Luciano Portela de Sousa

ORCID: https://orcid.org/0000-0002-0324-9499

Instituto Esperança de Ensino Superior, Brasil

E-mail: albinoportela@hotmail.com

\begin{abstract}
Resumo
Na obesidade, há um excesso de gordura, que podem ser localizadas ou não, e está condição pode acarretar prejuízos à saúde. Esse excesso leva a ocorrência de doenças cardiovasculares, diabetes, câncer, doenças renais. Para avaliar a sua ocorrência, este estudo avaliou questões relacionadas ao sobrepeso e obesidade em pessoas atendidas em uma Ação Integrada de Saúde (AIS), em três escolas municipais de Santarém-Pará. Trata-se de uma pesquisa descritiva, de abordagem quantitativa que seguiu o método descrito pelo Diagrama de Charles Maguerez, ocorrendo em cinco etapas: observação da realidade, pontos chave, teorização, hipótese de solução e aplicação à realidade. Os dados foram obtidos através de avaliação antropométrica, utilizando as variáveis: massa corpórea e altura, e aplicação de questionário socioeconômico. Oitenta e uma pessoas compuseram a amostra do estudo. Os resultados apontam que 32 pessoas apresentam sobrepeso, 30 obesidade, 1 baixo peso e $18 \mathrm{com}$ parâmetros normais, seno que a maioria dos pesquisados afirmaram possuir renda familiar de até dois salários-mínimos, correspondendo a $69.23 \%$ homens e $52.73 \%$ de mulheres Verifica-se que diante desses resultados devem-se se ter novos estudos que possam ampliar a amostra, e assim ter dados mais significativos sobre essa realidade, e a partir de tais informações possam ser incluídas no planejamento estratégico da atenção básica, ações preventivas, que contribuam na redução dos índices de sobrepeso e obesidade.
\end{abstract}

Palavras-chave: Sobrepeso; Obesidade; Epidemiologia; Saúde coletiva. 


\begin{abstract}
In obesity, there is an excess of fat, which can be localized or not, and this condition can cause damage to health. This excess leads to the occurrence of cardiovascular disease, diabetes, cancer, kidney disease. To assess its occurrence, this study evaluated issues related to overweight and obesity in people assisted by an Integrated Health Action (AIS) in three municipal schools in Santarém-Pará. This is a descriptive research, with a quantitative approach that followed the method described by Charles Maguerez's Diagram, taking place in five stages: observation of reality, key points, theorization, solution hypothesis and application to reality. Data were obtained through anthropometric assessment, using the variables: body mass and height, and application of a socioeconomic questionnaire. Eighty-one people comprised the study sample. The results show that 32 people are overweight, 30 obese, 1 underweight and 18 with normal parameters, whereas most respondents stated that they had a family income of up to two minimum wages, corresponding to $69.23 \%$ men and $52.73 \%$ women. given these results, new studies should be carried out that can expand the sample, and thus have more significant data on this reality, and from such information can be included in the strategic planning of primary care, preventive actions, which contribute to the reduction of overweight and obesity rates.
\end{abstract}

Keywords: Overweight; Obesity; Epidemiology; Public health.

\title{
Resumen
}

En la obesidad existe un exceso de grasa, que puede localizarse o no, y esta condición puede causar daños a la salud. Este exceso conduce a la aparición de enfermedades cardiovasculares, diabetes, cáncer, enfermedad renal. Para evaluar su ocurrencia, este estudio evaluó problemas relacionados con el sobrepeso y la obesidad en personas asistidas por una Acción Integrada de Salud (AIS) en tres escuelas municipales de Santarém-Pará. Se trata de una investigación descriptiva, con un enfoque cuantitativo que siguió el método descrito por el Diagrama de Charles Maguerez, que se desarrolla en cinco etapas: observación de la realidad, puntos clave, teorización, hipótesis de solución y aplicación a la realidad. Los datos se obtuvieron mediante evaluación antropométrica, utilizando las variables: masa corporal y altura, y aplicación de un cuestionario socioeconómico. Ochenta y una personas comprendieron la muestra del estudio. Los resultados muestran que 32 personas tienen sobrepeso, 30 obesidad, 1 bajo peso y 18 con parámetros normales, mientras que la mayoría de los encuestados afirma tener un ingreso familiar de hasta dos salarios mínimos, lo que corresponde al 69,23\% de hombres y 52,73\% de mujeres. , se deben realizar nuevos estudios que puedan ampliar la muestra, y así tener datos más significativos sobre esta realidad, y a partir de dicha información se puedan incluir en la planificación estratégica de la atención primaria, acciones preventivas, que contribuyan a la reducción del sobrepeso y la obesidad. tarifas.

Palabras clave: Sobrepeso; Obesidad; Epidemiología; Salud colectiva.

\section{Introdução}

A obesidade é uma condição crônica associada ao aumento de tecido adiposo, e em alguns casos ao aumento de massa muscular (Shen et al,2020), podendo ser prejudicial à saúde, já que pode levar a um desequilíbrio hormonal, distúrbio de autoimagem, associação a outras patologias (Neves et al, 2020). No que se refere a etiologia da obesidade, Wanderley e Ferreira (2010) afirmam que essa condição crônica resulta em prejuízos à saúde, a saber: desequilíbrio hormonal, dificuldade de realizar as suas tarefas diárias devido ao sedentarismo, distúrbios da autoimagem, o que pode levar a um quadro depressivo, entre outras condições.

$\mathrm{Na}$ literatura consta ainda, a escolaridade como determinante social importante para sua ocorrência, uma vez que esse fator, pressupõe á má condições de vida, pouca informação e orientação para redução de peso (Teichmann et al., 2006). Somase a isso, a baixa renda tendo em vista que a falta de recursos financeiros para o acesso a certos tipos de alimentos restringe sua alimentação a um cardápio mais calórico (Cavalcanti et al., 2007).

De acordo com Souza et al. (2018), o sobrepeso e a obesidade têm aumento nos últimos anos, o que gera certa preocupação, devido a sua associação a doenças crônicas, a exemplo da hipertensão e diabetes. De acordo com os parâmetros preconizados pela OMS para se determinar sobrepeso e obesidade utiliza-se o cálculo do IMC, baseado na divisão do peso pela estatura (Pitanga \& Lessa, 2005).

No Brasil, no ano de 2014, a Vigilância de Doenças Crônicas por Inquérito Telefônico (VIGITEL), realizou uma pesquisa com tamanho amostral de 40.853 pessoas, divulgada pela OMS, abrangendo as capitais e o Distrito Federal. Essa pesquisa demonstrou que mais da metade dessa população estava acima do peso cerca de 21.448 (52,5\%), sendo que deste 
total, 3.839 (17,9\%) apresenta obesidade. No ano de 2016, esse mesmo órgão fez uma pesquisa semelhante, desta vez utilizando uma amostra de 53.210, na qual foi constatado 28.626 (53,8\%) pessoas com excesso de peso. Analisando esses dados, principalmente, no que tange a Belém, houve uma adesão na pesquisa de 1.998 pessoas (735 homens e 1.263 mulheres), e o resultado mostrou que 55,6\% da amostra apresenta sobrepeso VIGITEL. (Brasil, 2015; Brasil, 2017)

Apesar desses dados, no estado do Pará, ainda há escassez de informações acerca dos fatores que tem levado a essa alta prevalência, e o quantitativo fora da região metropolitana de Belém, o que fomentou a proposição deste estudo com o objetivo de determinar a ocorrência de sobrepeso e obesidade associado aos determinantes sociais em saúde de pessoas atendidas na Ação Integrada de Saúde (AIS), em três escolas municipais de Santarém-Pará.

\section{Metodologia}

Trata-se de um estudo descritivo, transversal, de abordagem quali-quantitativo, baseado na Metodologia da Problematização (MP), usando como ponto de partida a realidade do sujeito, o cenário no qual está inserido e onde os vários problemas podem ser vistos, percebidos ou deduzidos, de maneira que possam ser estudados em conjunto ou em pares. (Evangelista, 2012)

Esta metodologia foi escolhida, por fazer parte do Projeto Pedagógico do Curso (PPC) de Enfermagem da UEPA, onde as metodologias ativas, auxiliam no aprendizado teórico prático das disciplinas, através do Diagrama de Charles Maguerez realizado em cinco etapas:

$1^{\text {a }}$ - Observação da realidade: Foi realizada uma pesquisa em três escolas de ensino fundamental: Frei Fabiano Merz, localizado na Av. Paulo Maranhão no bairro Caranazal, com o total de 59 entrevistados; Paulo Rodrigues dos Santos, localizado na Tv. Uirapuru no bairro Floresta, com 16 entrevistados; Maria Amália, localizada na R. Campos Sales no bairro Liberdade, com 6 entrevistados, no município de Santarém, situado no Baixo Amazonas, na região Oeste do Pará, observando possíveis problemáticas a serem estudadas nas instituições.

$2^{\mathrm{a}}$ - Pontos chave: Consistiu-se na identificação do problema. Assim, considera-se a população cada vez mais acima do peso, tornando-a um problema de saúde pública. A partir da observação de adultos aparentemente acima do peso foi definido o público-alvo, abrangendo funcionários e comunitários. Permaneceram incluídas na pesquisa as pessoas que aceitaram participar e autorizaram por meio do Termo de Consentimento Livre Esclarecido (TCLE), em conformidade com a Resolução no 466/12 do Ministério da Saúde, o uso dos resultados obtidos através do cálculo do IMC. Foram respeitados os aspectos éticos, para utilização dos dados levantados, com a aprovação do Comitê de Ética em Pesquisa (CEP) da UEPA Campus XII, conforme o parecer 1.318.965.

$3^{\mathrm{a}}$ - Teorização: Ocorreu por meio de pesquisa bibliográfica realizada com base em periódicos nacionais e internacionais, livros, teses e dissertações ao abordam assuntos relacionados à sobrepeso e obesidade. As plataformas informativas consultadas foram Scielo, Ministério da Saúde, Google Acadêmico, entre outros.

A partir do embasamento teórico, a pesquisa foi realizada baseada no critério de diagnóstico de obesidade a partir do IMC. Em razão disso, a coleta de dados foi realizada em dois momentos, primeiramente com a investigação do histórico clínico por meio de um questionário, contendo quatorze perguntas fechadas e objetivas com finalidade de verificar as condições de saúde e estilo de vida, e posteriormente houve avaliação antropométrica, utilizando balança digital e estadiômetro para análise dos dados.

De posse das medidas de massa corporal e estatura, foi calculado o IMC a partir do índice de Quetelet (Pitanga e Lessa, 2005), conforme a fórmula: [IMC = massa $(\mathrm{Kg}) /$ Estatura ao quadrado $(\mathrm{m})]$ e classificados com base nos parâmetros da OMS. 
Os dados obtidos foram lidos, observados e analisados utilizando os programas Word e Excel (Microsoft Office para Windows-2016) para produção textual, gráficos e tabelas.

$4^{\mathrm{a}}$ - Hipótese de solução: Consistiu-se em discussões com o intuito de encontrar alternativas para minimizar ou resolver o problema identificado. Dessa forma, foi decidido que seria realizada a aferição das medidas antropométricas para o cálculo do IMC, com orientações a partir dos resultados obtidos. Além disso, reconhecendo o potencial transformador da educação em saúde, foi acordado que seria efetivada uma palestra de cunho educativo acerca do problema de saúde pública que compõe a obesidade, seus fatores de risco e suas consequências para a qualidade de vida, bem como a importância da adoção de bons hábitos alimentares e a prática de atividade física.

$5^{\mathrm{a}}$ - Aplicação à realidade: Nessa etapa foram ministradas palestras para os participantes da pesquisa com o intuito de informar sobre as temáticas relacionadas á obesidade e a importância de práticas voltadas a qualidade de vida. Juntamente, foram entregues os resultados dos IMC, por profissionais da área da saúde, os quais orientaram os pesquisados individualmente.

\section{Resultados e Discussão}

Foram obtidos dados acerca do gênero, idade, escolaridade, renda familiar e classificação do IMC. Sendo o número da amostra composto por 81 pessoas, $55(67,90 \%)$ do gênero feminino, com idade média de 45 anos e $26(32,10 \%)$ do gênero masculino, com idade média de 46 anos.

Acerca do grau de escolaridade, os dados apontam que a maioria das mulheres $(52,73 \%)$ possuem ensino superior completo e apenas 3,64\% apresentam ensino fundamental. Isso se justifica devido a maior adesão das professoras, dentre todos os funcionários das escolas, pois essas necessitam do curso superior para exercer sua profissão. Entretanto, os resultados sobre escolaridade do gênero masculino foram diferentes do gênero feminino, prevalecendo o ensino fundamental $(23,08 \%)$ e a minoria no ensino superior $(15,38 \%)$, isso pode ser explicado de acordo com a entrevistas realizadas, pois a maioria declarou que trabalha em serviços gerais não necessitando de diploma de curso superior.

A escolaridade está relacionada com o nível de sobrepeso e obesidade, onde percebe-se que as pessoas com baixa escolaridade estão propensas ao excesso de peso (Teichmann et al, 2006). No entanto, ao avaliar as informações obtidas, em que os pesquisados são, em sua maioria, profissionais na área da educação, há uma contraposição com os resultados obtidos por esse autor, pois os entrevistados e sua maior parte têm sobrepeso ou obesidade. Logo, o excesso de peso em relação as pessoas com alta escolaridade pode estar relacionadas, provavelmente, a falta de tempo, prática de atividade física e má alimentação.

Consecutivamente, a maioria dos pesquisados afirmaram possuir renda familiar de até dois salários-mínimos, correspondendo a $69,23 \%$ homens e 52,73\% de mulheres. São classificadas como baixa renda, famílias com até três saláriosmínimos, classificação na qual se encaixa 58,02\% dos pesquisados (Kamakura et al, 2013). Dessa forma, é possível relacionar o perfil socioeconômico dos entrevistados com seus hábitos alimentares, que pessoas de baixa renda optam por comidas como arroz, feijão e carne, por serem consideradas mais "pesadas" trazendo a sensação de estômago cheio, o que as leva a desprezar folhas, frutas e legumes, por serem consideradas comidas leves (Romanelli, 2006). Porém, esse cardápio de alto valor energético exige também maior gasto de energia, através de atividade físicas de forma a evitar a obesidade.

Sobre a classificação do IMC uma quantidade significativa de mulheres e homens possuem sobrepeso, correspondendo a $40 \%$ e 38,56\%, respectivamente. A partir da categorização do IMC e a divisão por faixa etária, 62 indivíduos (76,54\%) são classificados com sobrepeso ou obesidade. O número de mulheres e homens com massa adiposa elevada está em maior ocorrência a partir dos 25 anos. Além disso, foi verificado que, em média, há dois homens e, aproximadamente, quatro mulheres com sobrepeso em cada faixa etária. Sob o mesmo ponto de vista, foi observado o maior quantitativo de obesidade 
grau I na faixa etária de 45-54 anos, encontrando-se 6 mulheres e 3 homens. Nesse mesmo intervalo de idade, 2 homens e 3 mulheres com obesidade grau II e na obesidade grau III é perceptível a ausência de casos do gênero masculino em comparação ao gênero feminino apresentando um caso aos 45 anos. (Tabela 1)

Tabela 1: Distribuição de sobrepeso e obesidade por gênero e faixa etária.

\begin{tabular}{ccccccccc}
\hline FAIXA ETÁRIA & SOBREPESO & \multicolumn{2}{l}{$\begin{array}{l}\text { OBESIDADE GRAU } \\
\text { I }\end{array}$} & $\begin{array}{l}\text { OBESIDADE } \\
\text { GRAU II }\end{array}$ & \multicolumn{2}{l}{$\begin{array}{l}\text { OBESIDADE } \\
\text { GRAU III }\end{array}$} \\
\hline & $M A S$. & $F E M$. & MAS. FEM. & MASC. FEM. & MASC. FEM. \\
$18-24$ & 1 & 1 & 0 & 0 & 0 & 0 & 0 & 0 \\
$25-34$ & 3 & 4 & 0 & 1 & 0 & 0 & 0 & 0 \\
$35-44$ & 2 & 8 & 1 & 4 & 1 & 0 & 0 & 0 \\
$45-54$ & 1 & 6 & 3 & 6 & 2 & 3 & 0 & 1 \\
$55-64$ & 3 & 2 & 1 & 3 & 2 & 0 & 0 & 0 \\
$\geq 65$ & 0 & 1 & 1 & 0 & 0 & 1 & 0 & 0 \\
\hline Total & 10 & 22 & 6 & 14 & 5 & 4 & 0 & 1 \\
\hline
\end{tabular}

Fonte: Autores.

Comparando as entrevistas realizadas pela VIGITEL nas capitais brasileiras e Distrito Federal em 2016, com 53.210 pessoas (20.258 homens e 32.952 mulheres) foi observado prevalência de sobrepeso e obesidade entre as faixas etárias de 55 a 64 anos. Ao fazer comparação com o vigente estudo é possível verificar a divergência em relação à idade, pois na faixa de 35 a 44 anos foi analisada a ocorrência do sobrepeso, enquanto a obesidade foi prevalente entre 45 a 54 anos. Quando tratado, apenas do sobrepeso das mulheres no estudo da VIGITEL, observa-se maior ocorrência na faixa entre 55 a 64 anos, enquanto na pesquisa realizada nas três escolas municipais era entre 35 a 44 anos. Porém essas diferenças podem ser decorrentes da grande desigualdade do número das amostras (Brasil, 2015; Brasil, 2017).

\section{Conclusão}

Os resultados desse estudo, permitiram avaliar os fatores comportamentais de risco para ocorrência da obesidade, demonstrando a importância da temática, já que tem aumentado os índices de pessoas sedentárias, com distúrbios alimentares, o que pode ocasionar o sobrepeso e consequente obesidade.

Sugere-se que deve ser realizado novos estudos, acerca das alterações de sobrepeso, visando o planejamento de ações para a redução dos riscos, como a educação em saúde acerca de uma alimentação equilibrada, prática esportiva, exames preventivos, para evitar complicações relacionadas a desordem fisiológica, gerada pelo consumo calórico exacerbado.

Em virtude de todo o exposto, é importante darmos ênfase ao papel do enfermeiro diante dessa problemática, tendo em vista que esse profissional é peça fundamental na atenção básica a saúde, que dentre inúmeros serviços faz parte da equipe que auxilia no tratamento de pessoas com sobrepeso e obesidade, realizando avaliações antropométricas e orientações na consulta de enfermagem e acompanhando o paciente na sua terapia. 
Research, Society and Development, v. 10, n. 9, e43110918208, 2021

(CC BY 4.0) | ISSN 2525-3409 | DOI: http://dx.doi.org/10.33448/rsd-v10i9.18208

\section{Referências}

Brasil (2015). Departamento de Análise de Situação de Saúde. Vigilância de Fatores de Risco e Proteção para Doenças Crônicas por Inquérito Telefônico. Brasília: DF.

Brasil (2017). Departamento de Análise de Situação de Saúde. Vigilância de Fatores de Risco e Proteção para Doenças Crônicas por Inquérito Telefônico, VIGITEL 2016. Brasília: DF.

Cavalcanti A. P. R., et al (2007) Crenças e influências sobre dietas de emagrecimento entre obesos de baixa renda. Ciênc. saúde coletiva.

Evangelista, I. A. S. (2012) Metodologia ativas: concepções e práticas. Revista em Foco; Ano 08; nº17

Fontelles, M. J.; et al (2009). Metodologia da pesquisa científica: diretrizes para a elaboração de um protocolo de pesquisa.

Francischi, R.P.P.; Pereira, L.O.; Freitas, C.S.; Klopfer, M.; Santos, R.C.; Vieira, P. et al. (2000) Obesidade: atualização sobre sua etiologia, morbidade e tratamento. Rev. Nutr., v. 13, n. 1, p. 17-28.

Jardim, Elisângela Duarte. (2014) Revisão de literatura-a temática da obesidade e a assistência de enfermagem frente a doença. 31 f. TCC (Graduação) - Curso de Enfermagem, Universidade Federal de Santa Catarina, Florianópolis.

Kamakura, Wagner Antonio; Mazzon, José Afonso. (2013). Estratificação socioeconômica e consumo no Brasil. Blucher.

Neves, S.C, Rodrigues, L.M, São Bento, P.A.S, Minayo, M.C.S. (2020). Os fatores de risco envolvidos na obesidade no adolescente: Uma revisão integrativa.. Cien Saude Colet [periódico na internet] .

Pitanga, Francisco José Gondim; Lessa, Ines (2005). Indicadores Antropométricos de Obesidade como Instrumento de Triagem para Risco Coronariano Elevado em Adultos na Cidade de Salvador - Bahia. Arquivos Brasileiros de Cardiologia, Bahia, v. 85, n. 1, p.26-31.

Romanelli, Geraldo. (2006) O significado da alimentação na família: Uma visão antropológica. Medicina, Ribeirão Preto, v. 3, n. 39, p.333-339.

Shen, J; Song, R; Ye, Y; Wu, X; Chow, W; Zhao, H. HIF3A (2020) DNA methylation, obesity and weight gain, and breast cancer risk among Mexican American women, Obesity Research \& Clinical Practice, Volume 14, Issue 6, Pages 548-553, https://doi.org/10.1016/j.orcp.2020.10.001

Souza, Saul de Azevêdo et al. (2018) Obesidade adulta nas nações: uma análise via modelos de regressão beta. Cadernos de Saúde Pública [online]. v. 34, n. 8, e00161417.

Teichmann, L et al. (2006) Fatores de risco associados ao sobrepeso e a obesidade em mulheres de São Leopoldo, RS. Revista Brasileira Epidemiol, São Leopoldo, v. 3, n. 9, p.360-373.

Wanderley Emanuela Nogueira, Ferreira Vanessa Alves (2010). Obesidade: uma perspectiva plural. Ciênc. saúde coletiva [Internet]; 15 ( 1 ): $185-194$. 